\title{
A Comprehensive Comparison of Period Extraction Algorithms for Asteroids with Long Term Observation
}

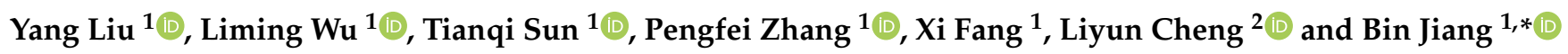 \\ 1 School of Mechanical, Electrical \& Information Engineering, Shandong University, Weihai 264209, China; \\ 201900800131@mail.sdu.edu.cn (Y.L.); 201900800512@mail.sdu.edu.cn (L.W.); \\ 201800600051@mail.sdu.edu.cn (T.S.); 201900800167@mail.sdu.edu.cn (P.Z.); fangxi@mail.sdu.edu.cn (X.F.) \\ 2 Institute of Computing Technology, Chinese Academy of Sciences, Beijing 100049, China; \\ chengliyun21@mails.ucas.ac.cn \\ * Correspondence: jiangbin@sdu.edu.cn
}

Citation: Liu, Y.; Wu, L.; Sun, T.;

Zhang, P.; Fang, X.; Cheng, L.; Jiang, B. A Comprehensive Comparison of Period Extraction Algorithms for Asteroids with Long Term Observation. Universe 2021, 7, 429. https://doi.org/10.3390/ universe7110429

Academic Editor: Jason P. Dworkin

Received: 16 October 2021

Accepted: 6 November 2021

Published: 10 November 2021

Publisher's Note: MDPI stays neutral with regard to jurisdictional claims in published maps and institutional affiliations.

Copyright: (c) 2021 by the authors. Licensee MDPI, Basel, Switzerland. This article is an open access article distributed under the terms and conditions of the Creative Commons Attribution (CC BY) license (https:// creativecommons.org/licenses/by/ $4.0 /)$.

\begin{abstract}
The light curve period of an asteroid plays an important role in determining the rotation period, the collision evolution and the YORP effect. There are many period extraction algorithms used to find the light curve period of asteroids with long term observation, which are mainly based on the frequency, time and time-frequency domains. This paper presents a comprehensive and unparalleled comparison of the popular algorithms based on the DAMIT (Database of Asteroid Models from Inversion Techniques) data set to show the statistical results. Considering the quoted period, absolute magnitude, diameter, albedo, time span and number of observations, we analyze the accuracy of five popular methods using the light curve data of 2902 asteroids. We find that although the performance of all the algorithms varies little, Phase Dispersion Minimization (PDM) performs better, followed by Lomb-Scargle (LS), while Conditional Entropy (CE) is not better than the others under certain conditions. We also analyze the cases which are more suitable for searching by frequencies or by periods.
\end{abstract}

Keywords: asteroids; general; methods; data analysis; techniques; photometric; astronomical databases; miscellaneous

\section{Introduction}

Most asteroids are solid and shine by reflecting sunlight, so the variation in the brightness can be observed under one or more of the following three conditions [1]:

(1) The Earth-asteroid and Sun-asteroid distance changes continuously.

(2) The irregular shape and the rotation of an asteroid.

(3) A change in the phase angle.

This brightness variation is called a light curve and the asteroid period can be extracted by measuring and analyzing light curves.

The determination of the light curve period of the asteroid plays an essential role in the subsequent study of asteroids. The light curve period indicates the time interval between two consecutive in-phase points on the light curve, that is, the brightness change period. It is one of the basic parameters of an asteroid, which is very close to its actual rotation period. There is a strong correlation between the light curve period and the rotation period, and the light curve period of an asteroid can be used to calculate its rotation period. Members of the asteroid family are regarded as fragments of huge asteroids formed after a catastrophic collision, and there is a clear relationship between the size of an asteroid and its period of rotation in accordance with the work of Dermott et al. [2]; accordingly, their rotation period distribution is of great significance for understanding the collision evolution of asteroids. Binzel [3] studied the rotational frequencies of two asteroid families (Eos Family, Koronis Family) and found that the distribution of the rotational frequencies differs widely between the two families, which can be explained as the two asteroid 
families underwent different collision evolutionary processes. Therefore, it is necessary to combine the geometric position as well as the shape model of the asteroid to fit the period. By recording and analyzing the time series of the light curve of a specific celestial body, the period can be found and verified, then the following observations would be organized in a targeted way [4]. In addition, Lowry et al. [5] reported that the asteroidal YORP (Yarkovsky-O'Keefe-Radzievskii-Paddack) effect is a torque that can modify the rotation rates and obliquities of small bodies in the solar system. Its causes are incident solar radiation pressure and the recoil effect from anistropic emission of thermal photons.

Butkiewicz-Bak et al. [6] proved that incomplete coverage of the rotation, noise or aliases stemming from gaps between separate light curves could lead to ambiguous periods for some asteroids. For example, asteroid (671) Carnegia has been observed many times and still requires further photometric monitoring to determine which period it belongs to [7]. However, there is little discussion in the literature of the role of period extraction algorithms in determining the periodicity of asteroids.

When it comes to algorithms for extracting the light curve period of the asteroid, there are several options. Szabó et al. [8] used the Lomb-Scargle (LS) method [9,10] to calculate the period of main-belt asteroids from the K2 mission; the Phase Dispersion Minimization (PDM) method [11] was used by Wang and Wang [12] to extract the periodicity of asteroid (1028) Lydina; asteroid (423) Diotima's rotation period was found by Prokof'eva and Karachkina [13] using the Jurkevich (JK) method [14], which is an analysis of the expected mean square deviation. Reshetnyk et al. [15] outlined that methods based on PDM, Fourier analysis and the Lomb normalized periodogram had similar results and higher accuracy. Sheppard et al. [16] argued that most astronomers used the Harris method [17], which is a Fourier analysis of light curves, to find the periods of asteroids. For instance, the determination of the periods of 23 main-belt asteroids was taken by the Harris method [18], and this method was applied to MPO (Minor Planet Observer) Canopus ${ }^{1}$ (a full-featured astrometry and photometry program capable of providing high accuracy results in both fields) by BDW Publishing (a website that provides MPO software) for the convenience of astronomers. However, the Fourier analysis-based approach is not suitable for the data set with inadequate time coverage [15] and the time complexity is high. The observed asteroids mentioned above are summarized in Table 1.

Table 1. Asteroids, algorithm, number of asteroids and reference of previous asteroid period calculation work.

\begin{tabular}{cccc}
\hline Asteroids & Algorithm & Number of Asteroids & Reference \\
\hline Main-belt asteroids from the K2 mission & LS & 955 & Szabó et al. [8] \\
asteroid (1028) Lydina & PDM & 1 & Wang and Wang [12] \\
Asteroid (423) Diotima & JK & 1 & Prokof'eva and Karachkina [13] \\
Main-belt asteroids & the Harris method & 23 & Riccioli et al. [18] \\
\hline
\end{tabular}

So far, some researchers, such as An et al. [4], have made contrasts of the effectiveness of different period extraction algorithms, and some of them used real variable star data while others used simulated data. Five methods were tested by Heck et al. [19] and it was found that none of these methods were distinctly better than the others. In addition, Swingler [20] proved that JK and PDM methods should be simply approximated as the Fourier method. The methods using phase binning such as the JK approach were generally less sensitive than those using smooth model functions such as LS [21]. Graham et al. [22] firstly used the real variable star data rather than simulated data to compare the popular period extraction algorithms and found that techniques based on the analysis of variance with harmonics and Conditional Entropy (CE) [23] consistently gave the best results. It was also argued that individual algorithms performed better than a simple ensemble approach.

Figure 1 shows the light curve of Asteroid No. 13, which we randomly selected from the DAMIT database. The abscissa is the time series of the asteroid, while the ordinate is brightness. Figure 2 depicts the effect of the five algorithms mentioned above to extract the light curve of Asteroid No. 13. The abscissa represents the different frequencies obtained 
by the extraction, and the ordinate is the power. The peak value of each graph represents the periodic component of the light curve extracted by this algorithm. It can be seen that, except for the frequency domain-based algorithm LS, the frequency of the asteroid's light variation period obtained by the other four algorithms are all $0.142 \mathrm{~h}^{-1}$, while the frequency obtained by the LS algorithm is $0.284 \mathrm{~h}^{-1}$. The main reason for this phenomenon is that in a light cycle, the light curve will mainly have two peaks, which are mainly related to the rotation of the asteroid and the two peaks are symmetrical. When the asteroid rotates half a circle or one circle, it will appear as a peak in the light curve. In the frequency-energy graph obtained by the LS algorithm, the 0.142 peak can also be seen. Therefore, the light curve measurement has a certain error, which should be $0.142 \mathrm{~h}^{-1}$. Furthermore, Harris et al. [24] have claimed that most asteroid lightcurves are dominated by the second harmonic of the rotation period, caused by elongated shape. However, if the shape is not very elongate, other harmonics may dominate, leading to the rotation period to be mainly affected by harmonics.

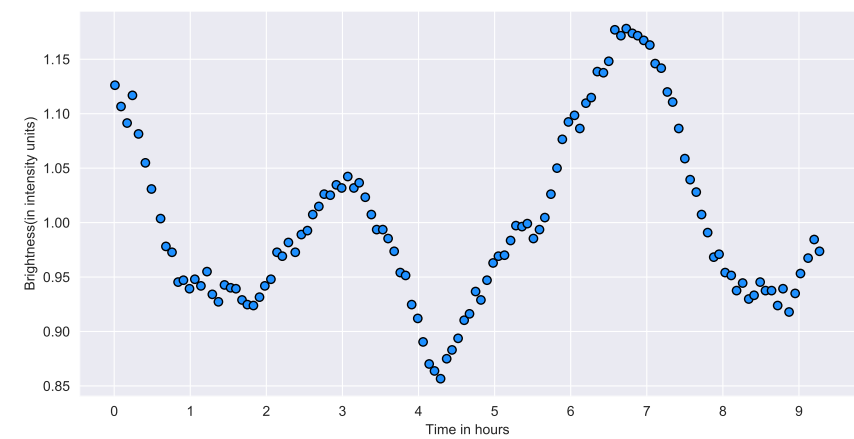

Figure 1. An example of the light curve of No. 13 asteroid (Egeria) in the DAMIT dataset.
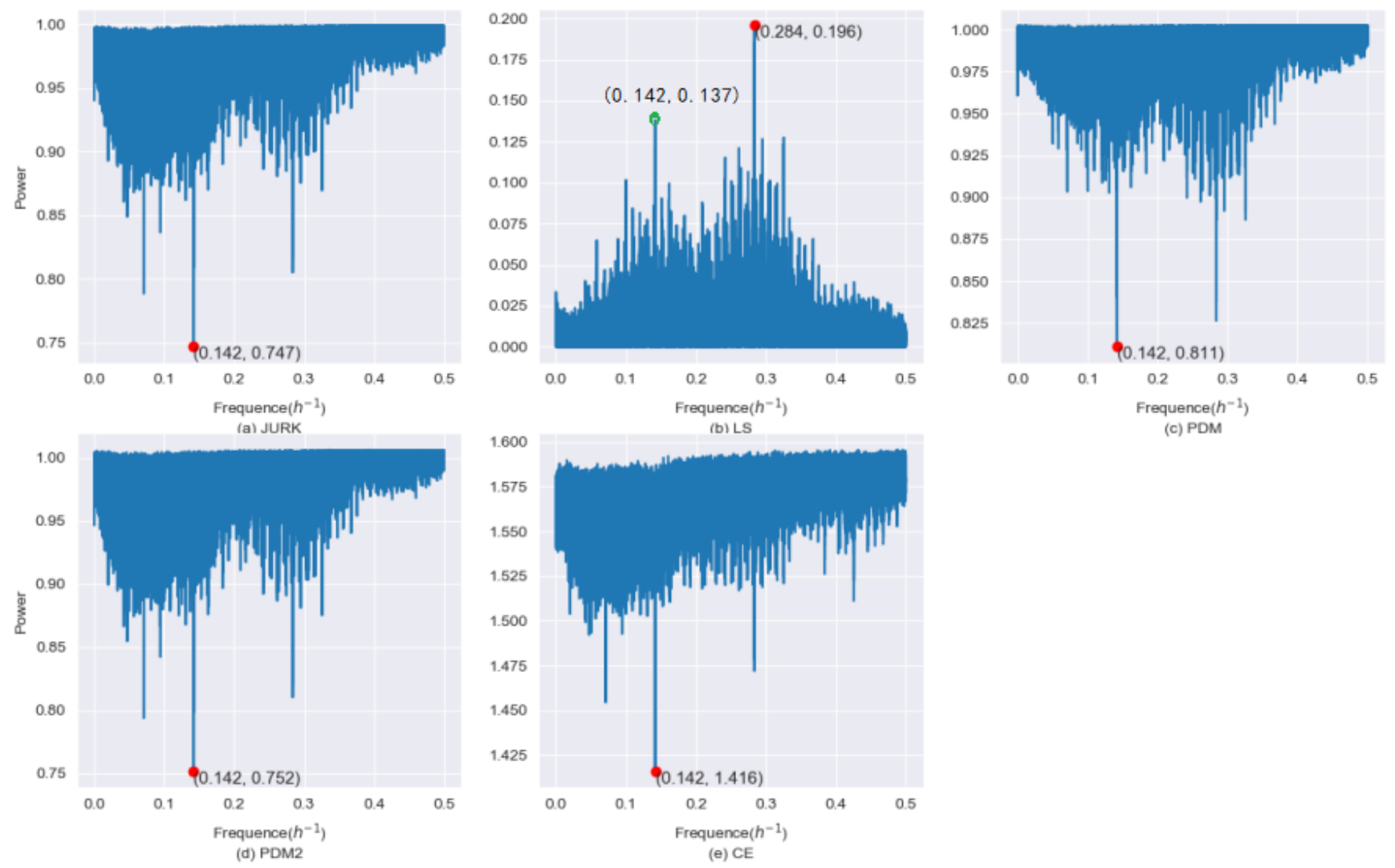

Figure 2. Taking No. 13 asteroid (Egeria) in the DAMIT dataset as an example. 
In our work, we used the real light curve data of asteroids to compare the effects of more common algorithms against different parameters of the observation and the object itself. This study will be useful for future period researches based on separate light curves of asteroids with long term observation. However, due to the data observation errors, our experimental results will inevitably have errors.

The article has been organized in the following way: Section 2 presents the information of the data set; Section 3 introduces some common period extraction algorithms; Section 4 explains the details of the selection of search steps and matching criteria; In Section 5, we specifically compare the extraction effect of five algorithms; Section 6 makes a further discussion on the five algorithms; Section 7 describes some related work, and Section 8 summarizes the article.

\section{Data Set}

DAMIT $^{2}$ [25] contains asteroid models derived using light curve inversion methods. By September 2021, the database contaied a total of 3304 asteroid data information. It contains a lot of parameter files about asteroids, such as the ecliptic latitude and longitude of the asteroid's autobiographical axis, rotation period, and other basic attributes.

Warner et al. [26] have been compiling asteroid lightcurve data in the Asteroid Lightcurve Database (LCDB) for more than 25 years with Warner and Pravec assisting the past several years. The main data contained in the LCDB are the light curve rotation period and amplitude, color index, H-G parameters, diameter, basic binary asteroid parameters, spin axis and shape model. By June 2021, the database contains data on 34,970 asteroids.

The light curve data we use is from DAMIT. Due to the longer time coverage and higher quality of the light curve, the period of the light curve is more reliable. For the study of various performances of the period extraction algorithms, in order to achieve the various characteristics of the light curve more clearly, we need the accuracy of the light curve to improve the reliability. It is a fact that the number of asteroids whose parameter records in LCDB are complete and which can be found in DAMIT is 2932. In order to make the data we obtain reliable, we have selected 2902 asteroids with a quality code rating $U \geq 2$ among the 2932 asteroids. The specific $U$ division basis can be seen in Section 2.1.

\subsection{The Quality Code Rating (U)}

The Asteroid Lightcurve Database (LCDB) ${ }^{3}$ established by Warner et al. [26] contains the quality code rating $(U)$ of the asteroid, which shows the accuracy of the reported period. The details of the specific evaluation levels are as shown in Table 2 and the $U$-distribution of 2932 asteroids is shown in Table 3.

Table 2. The current-day $U$ code assignment criteria [26].

\begin{aligned} & \hline$U$ Meaning \\ & \hline 0 Result later proven incorrect. This appears only on records of individual observations. \\ & \hline 1 Result based on fragmentary lightcurve(s), may be completely wrong. \\ & \hline $\begin{array}{l}\text { Result based on less than full coverage, so that the period may be wrong by } 30 \% \text { or so. } \\ \text { Also, a quality of } 2 \text { is used to note results where an ambiguity exists as to the number of } \\ \text { extrema per cycle or the number of elapsed cycles between lightcurves. Hence the result } \\ \text { may be wrong by an integer ratio. }\end{array} \\ &$\hline 3 Denotes a secure result with no ambiguity and full lightcurve coverage. \\ & \hline\end{aligned}


Table 3. The $U$-distribution of asteroids in DAMIT.

\begin{tabular}{ccc}
\hline$U$ & Number & Percentage \\
\hline 0 & 20 & $0.68 \%$ \\
1 & 13 & $0.44 \%$ \\
2 & 1286 & $43.86 \%$ \\
3 & 1613 & $55.02 \%$ \\
\hline
\end{tabular}

\subsection{Parameter Distribution}

In addition to $U$ of the asteroids, other information of the asteroids can also be obtained from LCDB and DAMIT. Therefore, we collected six parameters of each asteroid from the data sets, including the quoted period, absolute magnitude $(H)$, diameter, albedo, time span and number of observations. The probabilistic density distributions of the six are shown in Figure 3. Note that the periods of the asteroids are mainly between $2 \mathrm{~h}$ and $40 \mathrm{~h}$ with the minimum period being $2.13 \mathrm{~h}$, while there are also some longer periods. The absolute magnitude is mainly concentrated in 5-15 mag. It should also be noted that the number of sampling points varies greatly, which ranges from 51-23,970 with the time span up to $680,969.34 \mathrm{~h}(\sim 77.73 \mathrm{yr})$. Moreover, the albedo and diameter are primarily related to the class of the asteroid. The distributions of some parameters of the object itself in our data set are consistent with Warner et al. [26]. The median value of each parameter is summarized in Table 4.
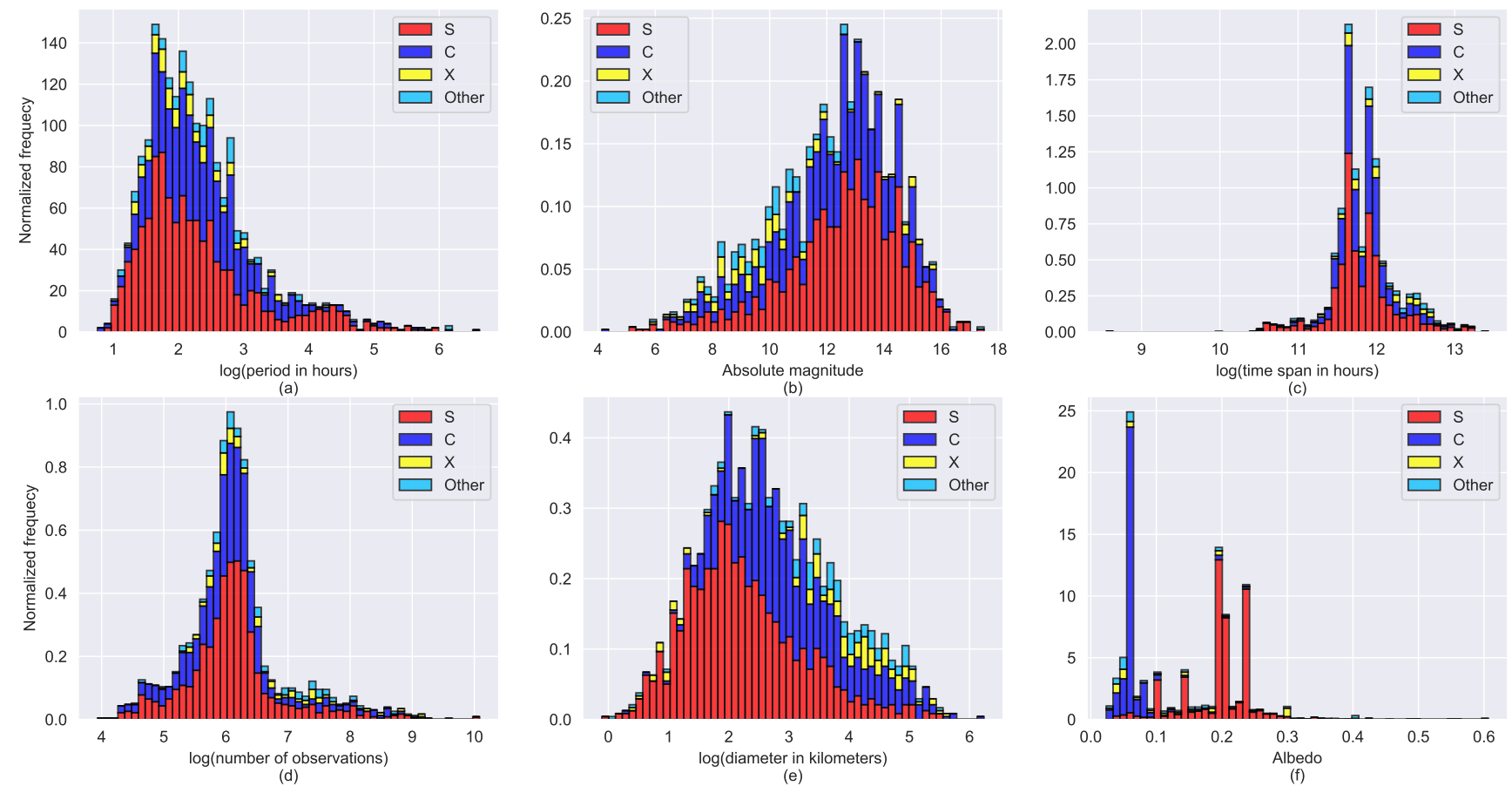

Figure 3. The distribution of (a) reported periods in hours, (b) absolute magnitudes, (c) time spans in hours, (d) number of points in each light curve, (e) diameters in kilometers and (f) albedos for asteroids of different classes in DAMIT. The "normalization" in the ordinate means: the integral of the probability density is 1 , that is, its total area (the sum of the products of the horizontal and vertical coordinates of each bar chart) is 1 . The letters in the legend indicate the codes of different types of asteroids classified by spectrum, which are mainly divided into S, C, X and Other types. Among them, S-type asteroids are stony meteorite asteroids, which account for $17 \%$ of all asteroids, and are the second most numerous asteroids, generally composed of silicides; C-type asteroids are carbonaceous chondrites. It accounts for $75 \%$ of all asteroids and is the most numerous asteroids, mostly distributed in the outer layer of the asteroid belt; X-type asteroids have very similar spectra, but there are still groups of different compositions (E-type, P-type, and P-type). In addition, other types of asteroids are relatively small, so they are unified into other types. In our data set, S-type asteroids account for a relatively high proportion of about $51.8 \%$, while C-type asteroids account for about $36.5 \%$, and X-type asteroids account for about $6 \%$. 
Table 4. Median value of each parameter for asteroids in DAMIT database.

\begin{tabular}{cccccc}
\hline Period (h) & H & Time Span (h) & Observations & Diameter (km) & Albedo \\
\hline 8.721 & 12.7 & 134611.385 & 450 & 12.55 & 0.157 \\
\hline
\end{tabular}

\section{Algorithms}

Section 3.1 discusses the details of the five algorithms mentioned above. Moreover, An et al. [4] have researched both advantages and disadvantages of these algorithms and we present part of the results in Sections 3.2 and 3.3.

\subsection{Details}

As for the algorithms, there are mainly three kinds of period extraction methods for unevenly sampled time series, which are based on the frequency domain, time domain and time-frequency domain respectively according to An et al. [4]. However, no algorithm is suitable for the periodic analysis of any type of non-uniform sampling sequence.

One of the most common methods based on the frequency domain is the LS periodogram method, which is modified from the Fourier spectral analysis via a least-squares fit. Date-Compensated Discrete Fourier Transform (DCDFT) [27] and CLEANest [28] algorithm are also used in some researches. The Harris method was developed specifically for the study of the light curve of the asteroid. Note that some signal features can be easily mined in the frequency domain, even if they are difficult to be detected in the time domain. However, when data points are few and the time span is large, the frequency resolution of the power spectrum is low.

The earlier analysis method based on the time domain is JK, a statistical algorithm for processing the irregularly sampled sequence based on the expected mean square error. Note that PDM is an extension of JK. In recent years, Graham et al. [23] put forward CE and applied it to variable star data which resulted in better performance. The main advantages of the time domain approaches are fast calculation and easy implementation, but the presence of periodic aliasing components will disturb the detection of other periodic components.

The dominant method in the time-frequency domain is the Weighted Wavelet Ztransform (WWZ) algorithm [29]. WWZ is a period extraction algorithm based on the wavelet analysis and vector projection. It is very suitable for the study of non-stationary signals and has advantages in the local characteristic analysis in terms of the time-frequency domain. The limits are the relatively long processing time and the complicated implementation.

According to the above methods, we selected five algorithms to compare the effects of various algorithms in terms of extracting the light curve period of asteroids, they are as follows:

(1) PDM, JK and LS algorithms that have been applied to the asteroid data, which we have mentioned in Section 1;

(2) PDM2 and CE algorithms that have been put forward in the last decade but have not been widely applied to the asteroid data.

Due to the high time complexity (In computer science, the time complexity is the computational complexity that describes the amount of computer time it takes to run an algorithm. Time complexity is commonly estimated by counting the number of elementary operations performed by the algorithm, supposing that each elementary operation takes a fixed amount of time to perform.), the Harris method and WWZ algorithm were not used in our research. The time complexity, parameters and references of each algorithm that we used are summarized in Table 5. The implementation was based on Python. 
Table 5. Theoretical time complexity, parameters and references of each algorithm.

\begin{tabular}{cccc}
\hline Algorithm & Time Complexity & Parameter & Reference \\
\hline Jurkevich (JK) & $O\left(n_{1} n_{2} N\right)$ & phase bins $=10$ & Jurkevich [14] \\
Lomb-Scargle (LS) & $O\left(n_{1} N\right)$ & & Lomb [9], Scargle [10] \\
Phase dispersion minimization (PDM) & $O\left(n_{1} n_{2} N\right)$ & phase bins = 5; covers per bin =2 & Stellingwerf [11] \\
Phase dispersion minimization (PDM2) & $O\left(n_{1} n_{2} N\right)$ & phase bins = 10 & Stellingwerf [30] \\
Conditional entropy (CE) & $O\left(n_{1} n_{2} N\right)$ & phase bins = 10; magnitude bins $=5$ & Graham et al. [23] \\
\hline
\end{tabular}

$n_{1}$ is the number of observations, $n_{2}$ is the number of bins and $N$ is the number of frequencies or periods.

\subsection{Advantages and Disadvantages of Period Extraction Algorithm Based on Frequency Domain (LS) Analysis}

The period extraction algorithms based on frequency domain analysis have two main advantages, which are as follows:

(1) A connection between time domain analysis and frequency domain analysis is built. Some signal features are difficult to detect in the time domain, but in In the frequency domain, it is easy to dig out.

(2) For time series with multi-period components, the analysis method based on frequency domain has obvious advantages in extraction.

At the same time, they also have the following shortcomings:

(1) When analyzing non-uniform time series with large time intervals, it is easy to produce harmonic interference, spectrum aliasing, etc. This phenomenon causes a large number of false peaks in the power spectrum. For example, when the LS algorithm analyzes time series with multi-period characteristics, it is easy to produce obvious false peaks in the power spectrum.

(2) When there are a few data points in the time series and a large time span, the frequency resolution of the power spectrum is low, and the reliable period range is narrow.

\subsection{Advantages and Disadvantages of Period Extraction Algorithm Based on Time Domain (JK PDM PDM2 CE) Analysis}

The advantages of the period extraction algorithms based on time domain analysis mainly have two points, which are as follows:

(1) These algorithms can avoid some problems found in traditional Fourier analysis methods, such as window and spectrum aliasing.

(2) There are fewer parameters that need to be defined in advance.

However, the methods based on time domain analysis also have shortcomings:

(1) The detection is limited to the time domain and lacks the ability to find hidden information in the frequency domain.

(2) When there are multi-period signal components in the time series, the analysis results of statistical methods are often complicated and confusing. It is difficult to separate the components of each cycle.

\section{Experiment}

\subsection{Data Preprocessing}

The light curve data of each asteroid consists of several blocks, and each block corresponds to different observers, telescopes or filters. The brightness is in intensity units with some being reduced to a unit distance from the Earth and the Sun when calibrated. We transformed the light-time from corrected JD epoch to hours. The solar phase angle where the asteroid was observed is one of the critical factors about the brightness so as to make a difference on the amplitude of the light curve [31]. Considering the phase angle difference in each block and the extreme variation of observation conditions between blocks, the brightness would be processed in the same way by using min-max to normalize if it meets the following conditions: 
(1) In the same block.

(2) The change of the phase angle is less than one degree.

Our experiment proved that this data preprocessing method could improve the accuracy of the periodic recovery.

\subsection{Searching Strategy}

Because the results may be affected by searching by frequencies or by periods, we used both the searching strategies and then compared the difference in the next Section.

\subsubsection{The Strategy of Frequency Searching}

Graham et al. [22] reported the Nyquist frequency:

$$
v_{N}=\frac{1}{2 \Delta T}
$$

where $\Delta T$ is the time interval. This is the maximum frequency of the searching range for an evenly sampled time series. McWhirter et al. [32] came up with Pseudo-Nyquist frequency, which is an upper limit for irregularly sampled data. He only changed the definition of $\Delta T$ to the mean time spacing between observations in Equation (1). In addition, the LombScargle class in the astropy package of Python $[33,34]$ used another method to define the maximum frequency:

$$
f_{\max }=\frac{x}{2} \times \frac{N}{\Delta t}
$$

where $x$ is a factor determined artificially, which is used to represent the maximum frequency of time series with non-equal intervals is greater than Nyquist frequency. $N$ is the number of points and $\Delta t$ is the time span. However, when we took the median value of time span in our data set as $\Delta t$, the median value of observations in our data set as $N$ and $0.5 \mathrm{~h}^{-1}$ as $f_{\text {max }}$ into Equation (2), we obtained $x=305$, which was too large for those asteroids that have shorter mean time spacings. We could fix the maximum frequency at $0.5 \mathrm{~h}^{-1}$ mainly for the following two reasons:

(1) According to the statistics of the rotation frequency of 2902 selected asteroids, it is found that the rotation frequency of all asteroids does not exceed $0.5 \mathrm{~h}^{-1}$.

(2) In addition, the rotation speed of large asteroids is very slow, and there is an upper limit to the speed. Few asteroids with a diameter greater than $100 \mathrm{~m}$ have a rotation period of less than $2.1 \mathrm{~h}$. Hartmann and Larson [35] have claimed that if the asteroid's rotation speed is faster than this speed, the inertial force on the surface is greater than the gravity, any loose surface material will be thrown out, and the asteroid may also disintegrate due to centrifugal force. Astronomers (quoted, $\mathrm{H}$ and L) generally believe that asteroids larger than $200 \mathrm{~m}$ are mainly composed of piles of gravel. Some of the smaller fragments thrown out may also become satellites of some asteroids. For example, Lin Shenxing (87 Sylvia) has two satellites. In our data set, the diameter of all asteroids is greater than $100 \mathrm{~m}$, and their rotation period is greater than $2.1 \mathrm{~h}$. Therefore, the maximum frequency will not exceed $0.5 \mathrm{~h}^{-1}$.

Vio et al. [36] found that the peak width of the correct frequency in the periodogram would be smaller when they used LS to process the light curve with a long timeline, which means that if the step size of the periodic search is too large, the frequency peak in the periodic graph might be missed. When processing large collections of time series, the following equation is often used as the step size setting [37]:

$$
f_{\text {step }}=\frac{1}{A \times \Delta t}
$$

where $A$ is a factor, typically 10 or 15 , and $\Delta t$ is the time duration. Debosscher et al. [38] and Richards et al. [39] both used $A=10$ in their works. However, McWhirter et al. [32] 
suggested this might be an overly fine frequency grid for their data set, as it seemed to produce peaks in noise.

We finally adopted $f_{\text {step }}$ defined in Equation (3) with $A=10, f_{\min }=0$ and $f_{\max }=0.5 \mathrm{~h}^{-1}$ to obtain the tested frequencies of each asteroid. The median time span in our data set corresponds to a median step size of $7 \times 10^{-7} \mathrm{~h}^{-1}$. In order to prevent the phenomenon reported by McWhirter et al. [32], we also set the frequency grids in $f_{\text {step }}=10^{-5} \mathrm{~h}^{-1}$ and $10^{-6} \mathrm{~h}^{-1}$ to make a comparison.

\subsubsection{The Strategy of Period Searching}

On condition of spending the same time on searching by periods as searching by frequencies at a fixed step size, the scope of searching by periods would be much smaller than searching by frequencies. The period is inversely proportional to the frequency. According to Figure 4, the periods of $92.3 \%$ asteroids are concentrated between $2 \mathrm{~h}$ and $40 \mathrm{~h}$. Due to the inverse relationship, the frequency changes in a periodic sequence spanning tens of hours. The value varies approximately between 0 and 0.5 , and since the period and frequency have a one-to-one correspondence, the value of frequency needs to be more refined.

For the step size, we chose three steps that have the similar computational time as searching by frequencies. They are $10^{-3} \mathrm{~h}$ with the searching range of $2-750 \mathrm{~h}, 10^{-4} \mathrm{~h}$ and $10^{-5} \mathrm{~h}$ with the searching range of $2-40 \mathrm{~h}$, respectively.

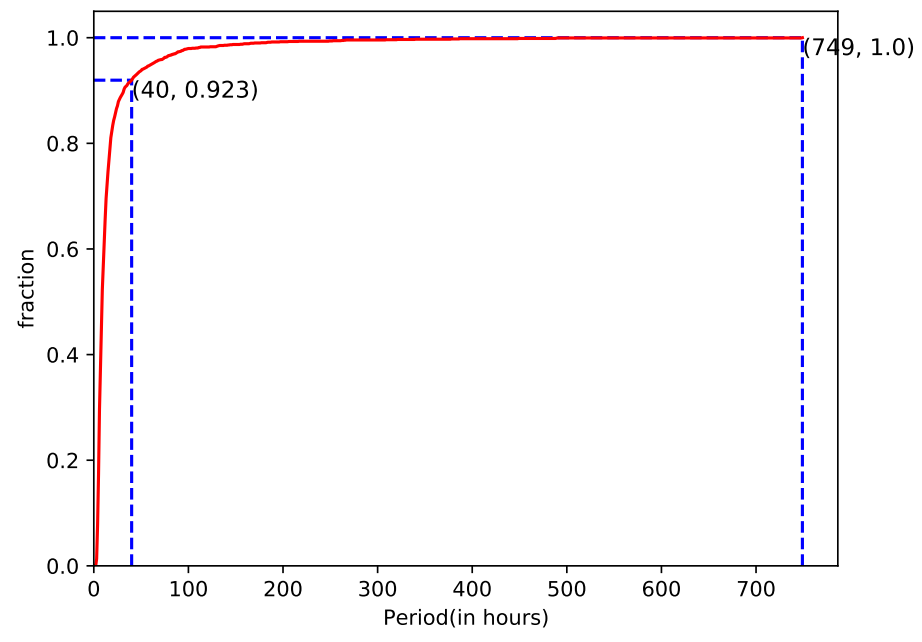

Figure 4. The cumulative reported period distribution of asteroids in our data set.

\subsection{Matching Criteria}

In order to determine whether the result calculated by the algorithm can successfully recover the reported period, it is necessary to determine a way to compare the reported and calculated period. Oluseyi et al. [40] firstly defined a metric describing whether the period is recovered or not when comparing the calculated and true period of the variable star:

$$
N \times \frac{\left|P_{c}-P_{r}\right|}{P_{r}} \leq \delta \phi_{\max }
$$

where $P_{r}$ is the reported period, $P_{c}$ is the period calculated by the algorithm, $N$ is the number of cycles defined as the ratio of the time span $\Delta t$ and the reported period $P_{r}$, and $\delta \phi_{\max }$ is the maximum allowed phase offset after period-folding $N$ cycles. This was translated alternatively to another way to express this measure for a $10 \mathrm{yr}$ baseline as:

$$
\frac{\left|P_{c}-P_{r}\right|}{P_{r}^{2}} \leq 10^{-5} d^{-1}
$$


Graham et al. [22] also summarized that Dubath et al. [41] and Richards et al. [39] used an accuracy level of $10^{-4} \mathrm{~d}^{-1}$ with $\delta \phi_{\max }=10 / 27$ and that of $10^{-3} \mathrm{~d}^{-1}$ with $\delta \phi_{\max }=100 / 27$, respectively. Graham et al. [22] finally adopted the above three equivalent accuracy cutoffs of $10^{-3} \mathrm{~d}^{-1}, 10^{-4} \mathrm{~d}^{-1}$ and $10^{-5} \mathrm{~d}^{-1}$ for a $10 \mathrm{y}$ baseline to carry out a comparison experiment of period extraction algorithms for variable star data. Due to the phenomenon that period harmonics often occur in the light curve of a variable star, the formula was modified to make sure that the periodicity could still be detected even though a harmonic of the reported period was found. The modified metric is as follows:

$$
\begin{aligned}
& \left|\frac{P_{c}}{P_{r}}-\left\|\frac{P_{c}}{P_{r}}\right\|\right|<\frac{\delta \phi_{\max } P_{r}}{\Delta t} \text { for } P_{c}>P_{r} \\
& \left|\frac{P_{r}}{P_{c}}-\left\|\frac{P_{r}}{P_{c}}\right\|\right|<\frac{\delta \phi_{\max } P_{r}}{\Delta t} \text { for } P_{c}<P_{r}
\end{aligned}
$$

where $\|x\|$ is the nearest integer to $x$. Considering that the harmonics also appear in the light curves of most asteroids [24], we finally adopted Equation (6) as the metric in our experiments. For each algorithm, only the frequency or period corresponding to the most apparent peak was selected as $P_{\mathcal{c}}$, so as to ensure the fairness when comparing the effect of each algorithm.

Because the light curve data of asteroids differs widely by time span, fixing $\delta \phi_{\text {max }}$ but not accuracy level as the cutoff is more suitable for our data set. We obtained $\delta \phi_{\max }=$ $8500 / 50,850 / 50$ and $85 / 50$ corresponding to accuracy cutoffs of $10^{-3} \mathrm{~h}^{-1}, 10^{-4} \mathrm{~h}^{-1}$ and $10^{-5} \mathrm{~h}^{-1}$ for the median time span in our data set and we used these three $\delta \phi_{\text {max }}$ in the Equation (6) in the next Section.

\section{Results and Analysis}

The physical parameters of asteroids and the quality of light curves have a great impact on the results, so we consider the parameters mentioned in Section 2.2 when comparing the results of the algorithms. In addition, whether to use period or frequency searching to scan and the step size of scanning also have a certain influence on the results.

\subsection{Absolute Magnitude}

Figure 5 shows the fraction of asteroids having a successful periodic recovery at different magnitudes. There is a general trend that as the magnitude increases, the proportion decreases. This shows that absolute magnitude is not conducive to period extraction to some extent. It can be noted that the effect of each algorithm varies little at brighter magnitudes. However, at faint magnitudes, the performance of CE is not comparatively better than the other algorithms.
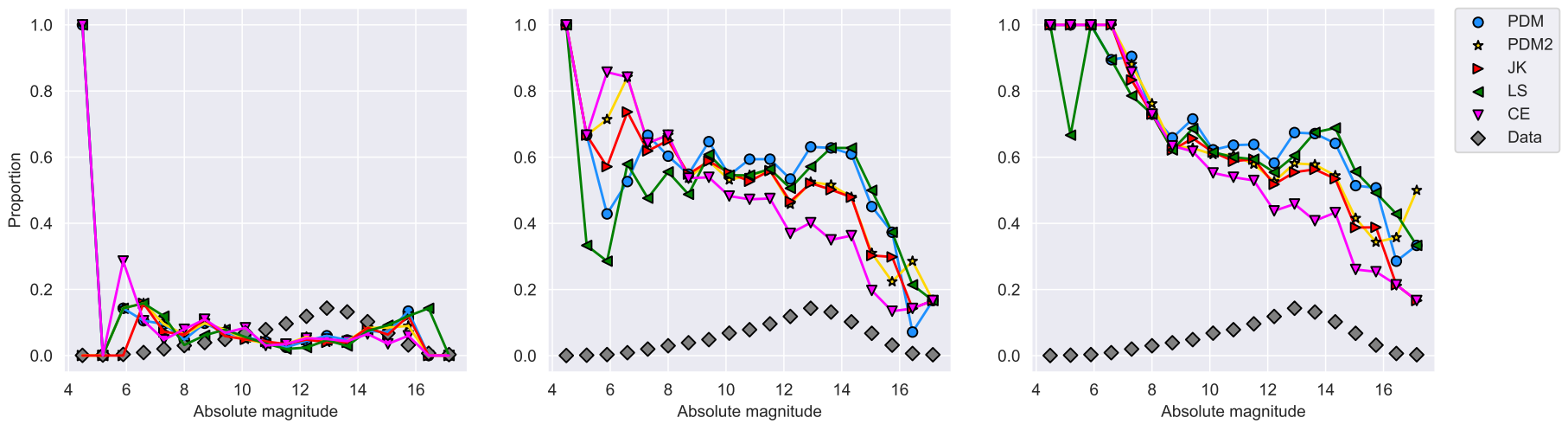

Figure 5. The comparison of different period finding algorithms considering the different absolute magnitudes for the step size defined in Equation (3) with $A=10$. The criterion for comparison is the proportion of asteroids having a successful periodic recovery. From left to right, the three plots are for different $\delta \phi_{\max }=85 / 50,850 / 50$ and $8500 / 50$. The gray diamonds indicate the proportion of asteroids at the corresponding absolute magnitude in our data set. 


\subsection{Observations}

Figure 6 shows the proportion of asteroids having a successful periodic recovery at different number of observations. It can be seen that the effects of various algorithms are not strongly dependent on the number of sample points when the number is greater than 1000. This shows that the number of observations can affect the lower limit of period extraction, but not the upper limit. However, the results of PDM and LS are better when the number of sample points is less than 1000 .
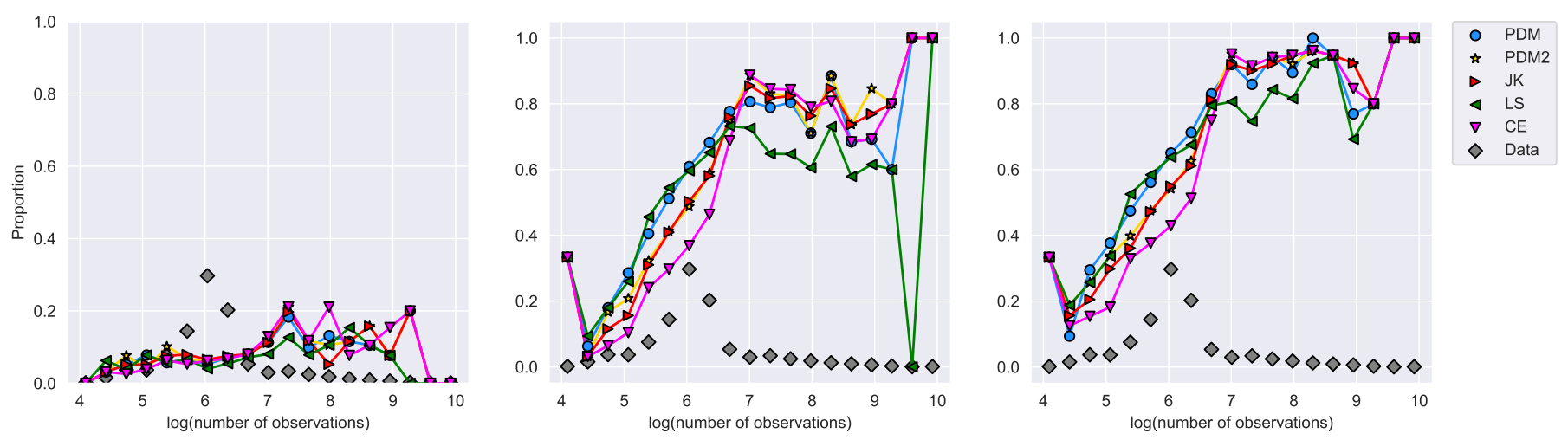

Figure 6. The comparison of different period finding algorithms considering the different number of observations for the step size defined in Equation (3) with $A=10$. The criterion for comparison is the proportion of asteroids having a successful periodic recovery. From left to right, the three plots are for different $\delta \phi_{\max }=85 / 50,850 / 50$ and $8500 / 50$. The gray diamonds indicate the proportion of asteroids at the corresponding number of observations in our data set.

\subsection{Time Span}

When studying variable stars, Polakis [42] reported that the longer time range of data was of greater value to eliminate any ambiguity in the period spectrum and greatly improved the accuracy of the calculated period. Figure 7 proves that this phenomenon also exists in the light curve of the asteroid. When $\delta \phi_{\max }=8500 / 50$, the proportion of asteroids having a successful recovery is positively correlated with the time span.
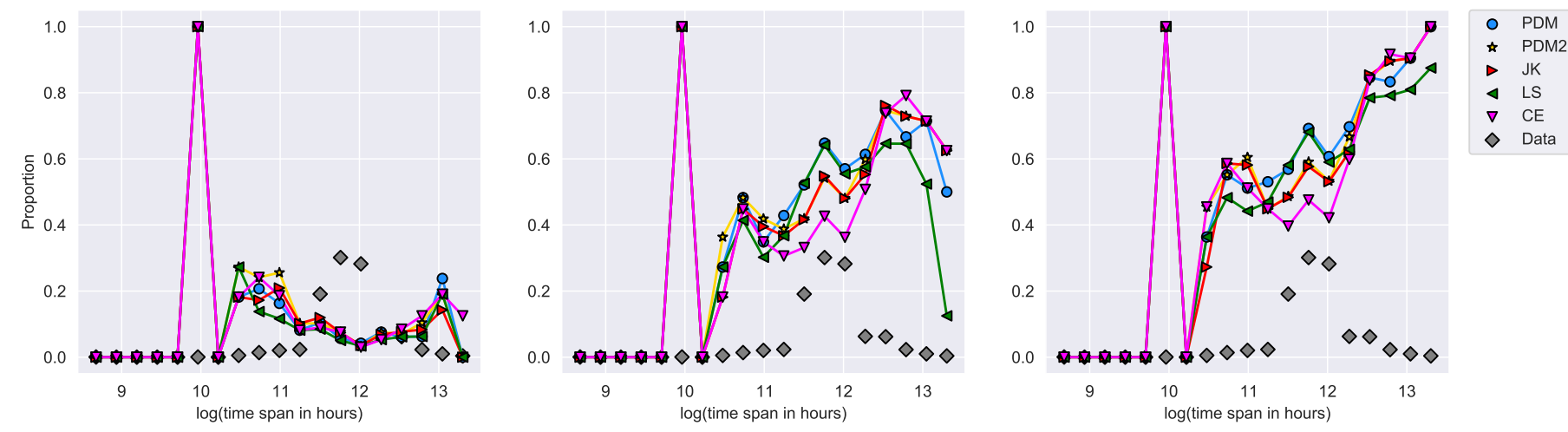

Figure 7. The comparison of different period finding algorithms considering the different time spans for the step size defined in Equation (3) with $A=10$. The criterion for comparison is the proportion of asteroids having a successful periodic recovery. From left to right, the three plots are for different $\delta \phi_{\max }=85 / 50,850 / 50$ and $8500 / 50$. The gray diamonds indicate the proportion of asteroids at the corresponding time span in our data set.

\subsection{Albedo}

In Figure 8, it is found that when the albedo is less than 0.3, there is little dependence on the albedo; when the albedo is greater than 0.3 , the result has a large fluctuation. The number of asteroids with albedo greater than 0.3 in our data set is found to be only 108 as- 
teroids, accounting for about $3.68 \%$, so when the albedo is greater than 0.3 , the periodic extraction effect has greater chance.
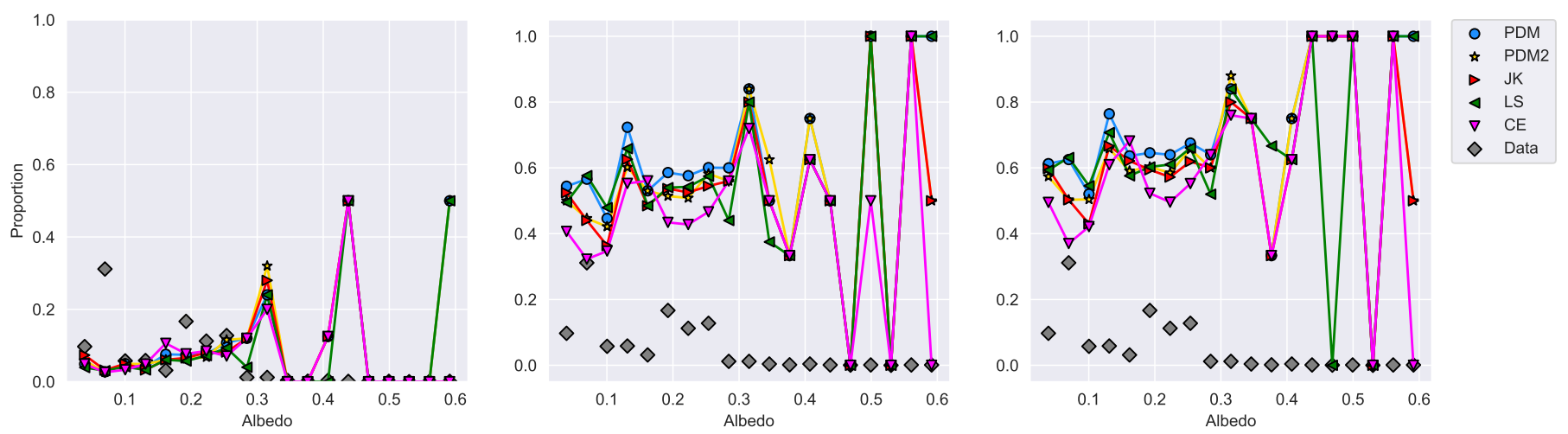

Figure 8. The comparison of different period finding algorithms considering the different albedos for the step size defined in Equation (3) with $A=10$. The criterion for comparison is the proportion of asteroids having a successful periodic recovery. From left to right, the three plots are for different $\delta \phi_{\max }=85 / 50,850 / 50$ and $8500 / 50$. The gray diamonds indicate the proportion of asteroids at the corresponding albedo in our data set.

\subsection{Diameter}

In terms of the diameter of the asteroid, Figure 9 shows that when the diameter is between $2 \mathrm{~km}$ to $148 \mathrm{~km}$, there is not a significant fluctuation on the effects of various algorithms. However, when the diameter is larger than $148 \mathrm{~km}$, the proportion of asteroids having a successful periodic recovery shows an increasing trend with the increase of the diameter, and the differences among various algorithms not appearing to be conspicuous. Therefore, when the diameter is larger than $148 \mathrm{~km}$, these algorithms are all acceptable choices. However, relatively speaking, the LS and PDM methods perform a little better in these methods.
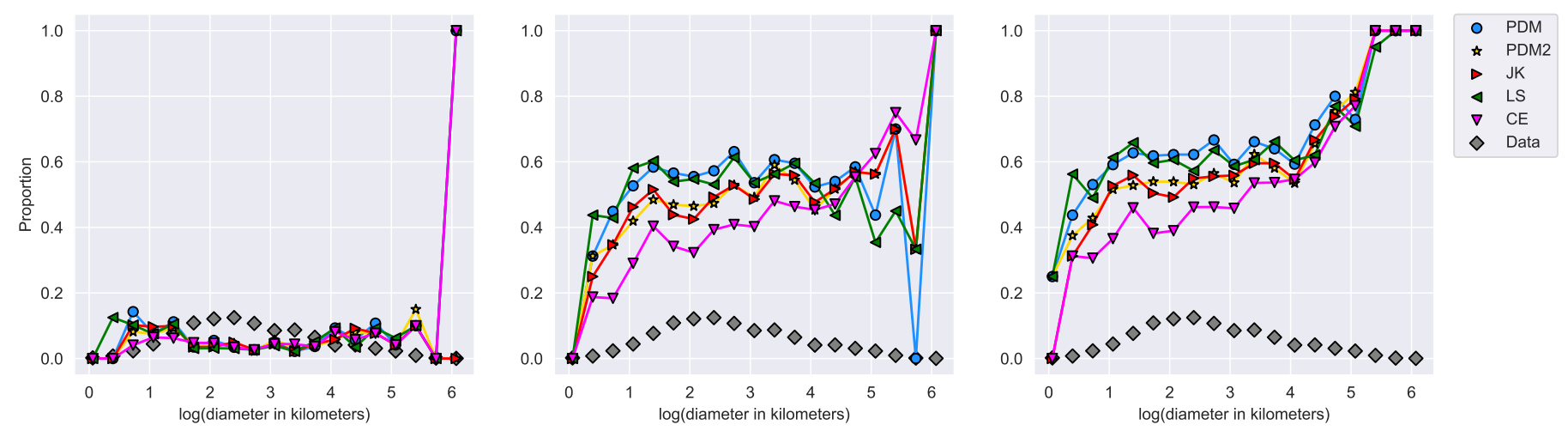

Figure 9. The comparison of different period finding algorithms considering the different diameters for the step size defined in Equation (3) with $A=10$. The criterion for comparison is the proportion of asteroids having a successful periodic recovery. From left to right, the three plots are for different $\delta \phi_{\max }=85 / 50,850 / 50$ and $8500 / 50$. The gray diamonds indicate the proportion of asteroids at the corresponding diameter in our data set.

\subsection{Step Size}

Different searching step size effects the accuracy and time complexity, so we integrated all the asteroids in our data set to calculate the proportion of those asteroids having a successful periodic recovery in terms of different step sizes for each algorithm. Here, we chose to use frequency searching strategy and we also used $\phi_{\max }=8500 / 50$ as the matching criteria to show the difference more clearly, the comparison results are shown in Table 6. It should be noticed that for all algorithms the results of $f_{\text {step }}=10^{-6} \mathrm{~h}^{-1}$ and 
$f_{\text {step }}=$ ste $_{\text {std }}$ vary very little. However, ste $p_{\text {std }}$ causes the longer time of calculation, this is because the median step size is $7 \times 10^{-7} \mathrm{~h}^{-1}$, which we have explained in Section 4.2.1. PDM performs better in all the search grids, followed by LS, while CE is not better than the others under certain conditions.

Table 6. The proportion of asteroids having a successful periodic recovery for different period finding algorithms using different frequency search grids.

\begin{tabular}{cccccc}
\hline$f_{\text {step }}$ & PDM & PDM2 & JK & LS & CE \\
\hline $10^{-5} \mathrm{~h}^{-1}$ & 0.500 & 0.490 & 0.447 & 0.503 & 0.371 \\
$10^{-6} \mathrm{~h}^{-1}$ & 0.633 & 0.561 & 0.554 & 0.612 & 0.465 \\
step $p_{\text {std }}$ & 0.632 & 0.560 & 0.554 & 0.613 & 0.467 \\
\hline
\end{tabular}

step $p_{\text {std }}$ means the $f_{\text {step }}$ defined in Equation (3) with $A=10$.

\subsection{Searching by Periods or by Frequencies}

Firstly, in order to make the working principle of the algorithms clearer, here we take the JK algorithm to extract the photometric curve period as an example:

(1) Find the variance $S$ of all the observed brightness of the asteroid.

(2) Assuming the test period T1, divide the observation time s of the light curve by $T 1$, the decimal place is the phase, and the phase data distribution is $[0,1)$.

(3) Divide the phase data into several segments, and calculate the variance of the brightness in each segment.

(4) Calculate the average value s of the above variance.

(5) The result corresponding to the given test accuracy and test period: $J=s / S$.

(6) Make $T 1=T 1+\Delta T$ ( $\Delta T$ is the step we choose), and repeat steps $2-6$ until we get the minimum $J$ within the error range. Then, $T 1$ is what we want.

Table 7 displays the period range corresponding to the period step size of different orders of magnitude after converting frequencies $\left(f_{\text {step }}=10^{-6} \mathrm{~h}^{-1}\right.$, ranging from 0 to $0.5 \mathrm{~h}^{-1}$ ) to periods. The total number of frequency searches is $0.5 / 10^{-6}=5 \times 10^{5}$, and that is approximately $(40-2) / 10^{-4}=3.8 \times 10^{5}$ when searching by periods $\left(p_{\text {step }}=10^{-4} \mathrm{~h}\right.$, ranging from $2 \mathrm{~h}$ to $40 \mathrm{~h}$ ). Although the order of magnitude of searching by frequencies is about the same as that of searching by periods, searching by frequencies performs better than by periods for each algorithm when the reported period is less than $10 \mathrm{~h}$ (see Figures 10 and 11). Because the maximum value of searching by periods is limited to $40 \mathrm{~h}$, the performance shows a 'cliff-like drop' if the reported period is more than $40 \mathrm{~h}$ (the recovery of the effect at longer periods when $\phi_{\max }=8500 / 50$ is due to the consideration of the harmonic problem). This problem also occurs in searching by periods having the step size of $10^{-5} \mathrm{~h}$, the effect of which within $10 \mathrm{~h}$ is comparable to that of searching by frequencies (see Figure 12). In Figure 13, we expanded our period searching range to $2-750 \mathrm{~h}$ and selected $10^{-3} \mathrm{~h}$ as the step size. This strategy has a similar order of magnitude as that for searching by frequencies. With this condition, it is clear that the effect of searching by periods is comparable to that of searching by frequencies at longer periods, however this searching strategy works very poorly at short periods.

Table 7. The maximum and minimum periods corresponding to the period step size of different orders of magnitude after converting the frequencies $\left(f_{\text {step }}=10^{-6} \mathrm{~h}^{-1}\right.$, ranging from 0 to $\left.0.5 \mathrm{~h}^{-1}\right)$ to periods.

\begin{tabular}{ccc}
\hline$p_{\text {step }}$ & $p_{\min }$ & $p_{\text {max }}$ \\
\hline $10^{-6}$ & $2 \mathrm{~h}$ & $3.16 \mathrm{~h}$ \\
$10^{-5}$ & $3.16 \mathrm{~h}$ & $10 \mathrm{~h}$ \\
$10^{-4}$ & $10 \mathrm{~h}$ & $31.6 \mathrm{~h}$ \\
$10^{-3}$ & $31.6 \mathrm{~h}$ & $100 \mathrm{~h}$ \\
$10^{-2}$ & $100 \mathrm{~h}$ & $316.2 \mathrm{~h}$ \\
$10^{-1}$ & $316.2 \mathrm{~h}$ & $1000 \mathrm{~h}$ \\
\hline
\end{tabular}



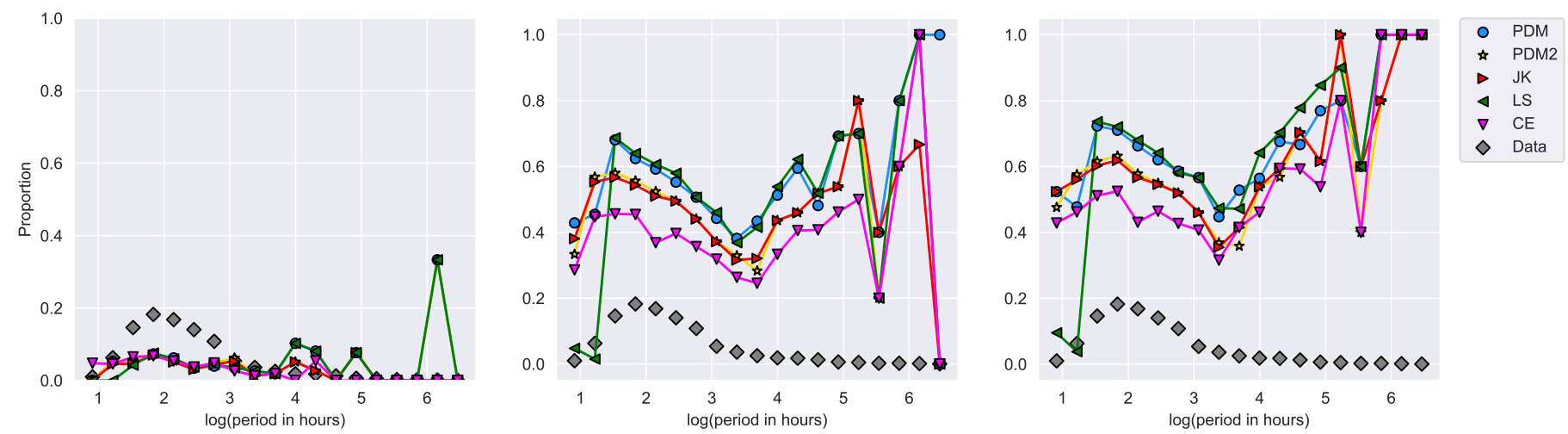

Figure 10. The comparison of different period finding algorithms considering the different reported periods for the step size of $10^{-6} \mathrm{~h}^{-1}$ of frequency searching. The criterion for comparison is the proportion of asteroids having a successful periodic recovery. From left to right, the three plots are for different $\delta \phi_{\max }=85 / 50,850 / 50$ and $8500 / 50$. The gray diamonds indicate the proportion of asteroids at the corresponding reported period in our data set.
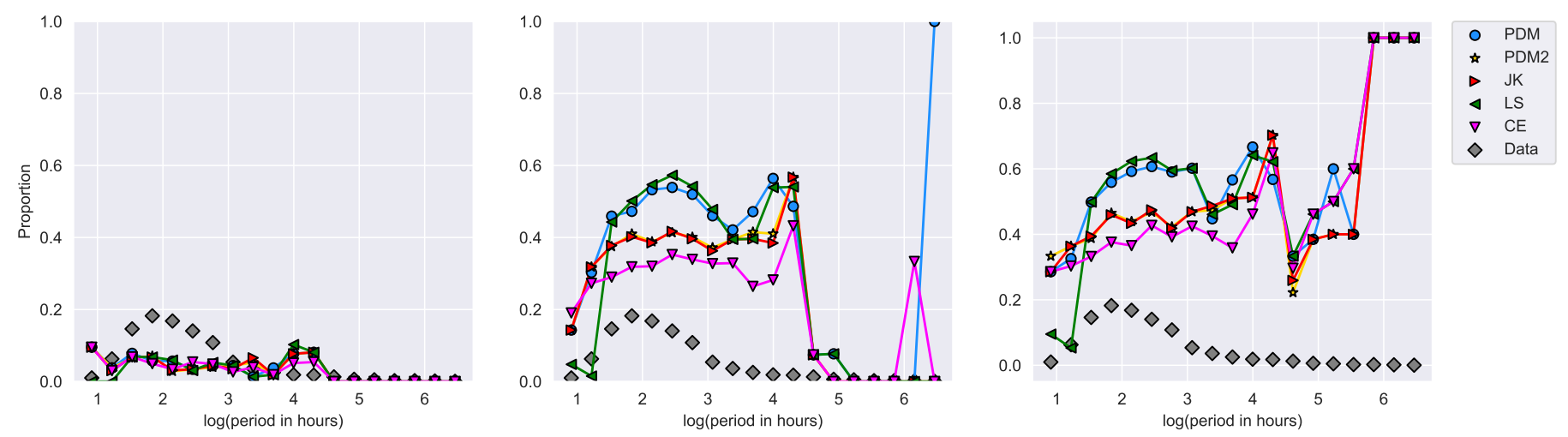

Figure 11. The comparison of different period finding algorithms considering the different reported periods for the step size of $10^{-4} \mathrm{~h}$ and the range of $2 \mathrm{~h}-40 \mathrm{~h}$ of period searching. The criterion for comparison is the proportion of asteroids having a successful periodic recovery. From left to right, the three plots are for different $\delta \phi_{\max }=85 / 50,850 / 50$ and $8500 / 50$. The gray diamonds indicate the proportion of asteroids at the corresponding reported period in our data set.
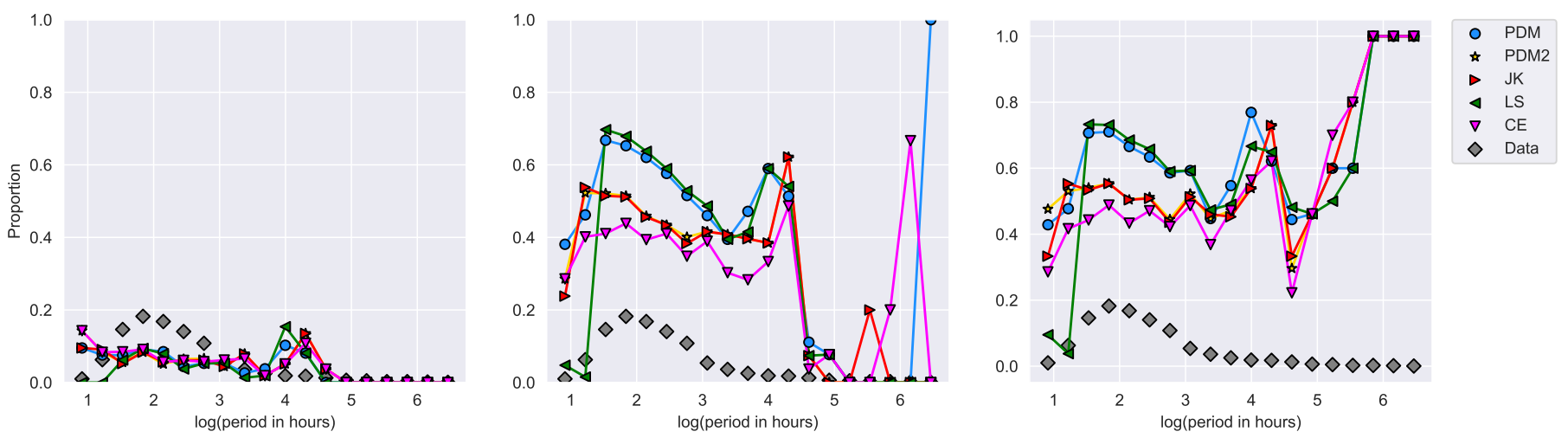

Figure 12. The comparison of different period finding algorithms considering the different reported periods for the step size of $10^{-5} \mathrm{~h}$ and the range of $2 \mathrm{~h}-40 \mathrm{~h}$ of period searching. The criterion for comparison is the proportion of asteroids having a successful periodic recovery. From left to right, the three plots are for different $\delta \phi_{\max }=85 / 50,850 / 50$ and $8500 / 50$. The gray diamonds indicate the proportion of asteroids at the corresponding reported period in our data set. 

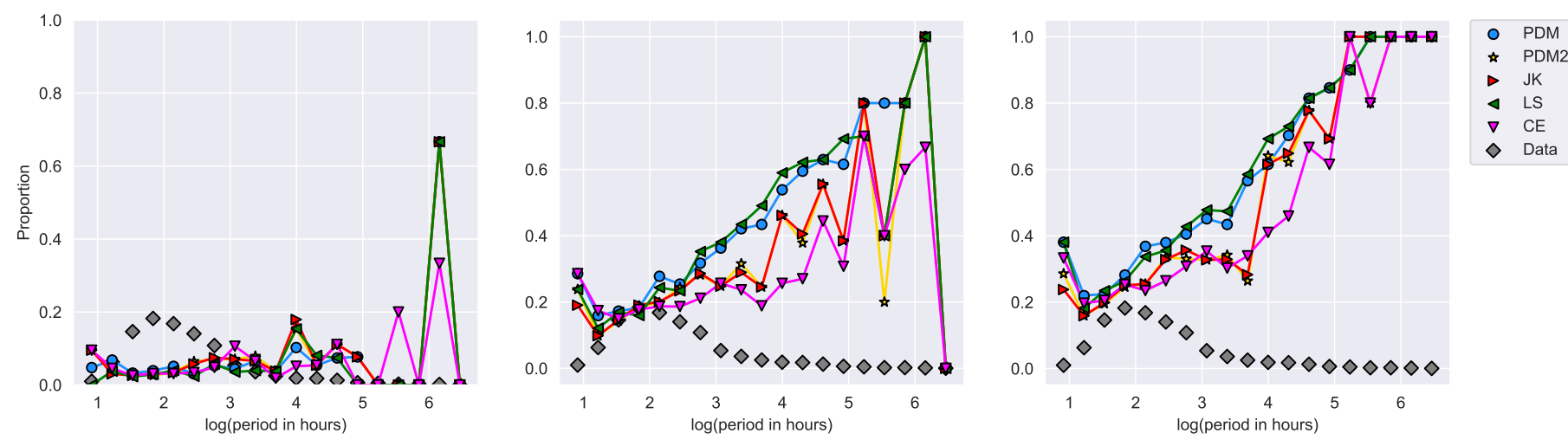

Figure 13. The comparison of different period finding algorithms considering the different reported periods for the step size of $10^{-3} \mathrm{~h}$ of and the range of $2 \mathrm{~h}-750 \mathrm{~h}$ of period searching. The criterion for comparison is the proportion of asteroids having a successful periodic recovery. From left to right, the three plots are for different $\delta \phi_{\max }=85 / 50,850 / 50$ and $8500 / 50$. The gray diamonds indicate the proportion of asteroids at the corresponding reported period in our data set.

\subsection{Time Performance}

In Table 5, we summarized the theoretical time complexity and parameters of each algorithm, which are also important for processing multiple batches of asteroid data. For each algorithm, we separately measured the time of computation in terms of searching by frequencies $\left(f_{\text {step }}=10^{-6} \mathrm{~h}^{-1}\right.$, ranging from 0 to $\left.0.5 \mathrm{~h}^{-1}\right)$ and searching by periods $\left(p_{\text {step }}=10^{-4} \mathrm{~h}\right.$, ranging from 2 to $40 \mathrm{~h}$ ) on the machine (a server with a $2.0 \mathrm{GHz}$ Intel Xeon e5 CPU and 56 virtual processors). The technology we took is multiple processes that could deal with data from up to 45 asteroids at the same time in Python. To show the difference of the processing time required for each algorithm, we selected three asteroids as examples, namely Olympiada (1022) having the median number of points, i.e., 441, Gretia (984) having 1510 points, and Wakkanai (5960) having 102 points, respectively. In Figure 14, The calculation time of each algorithm is reflected by the depth of color. The darker the color in figure, the shorter the calculation time. The average extraction time of the $\mathrm{CE}$ algorithm is greater than about $300 \mathrm{~s}$, the average extraction time of the PDM $\backslash \mathrm{PDM} 2 \backslash \mathrm{JK}$ algorithm is about $180 \mathrm{~s}-240 \mathrm{~s}$, and the LS algorithm is a search algorithm based on the frequency domain, and the average extraction time is the shortest (around $60 \mathrm{~s}$ ), accounting for $1 / 5$ of the $C E$ algorithm.

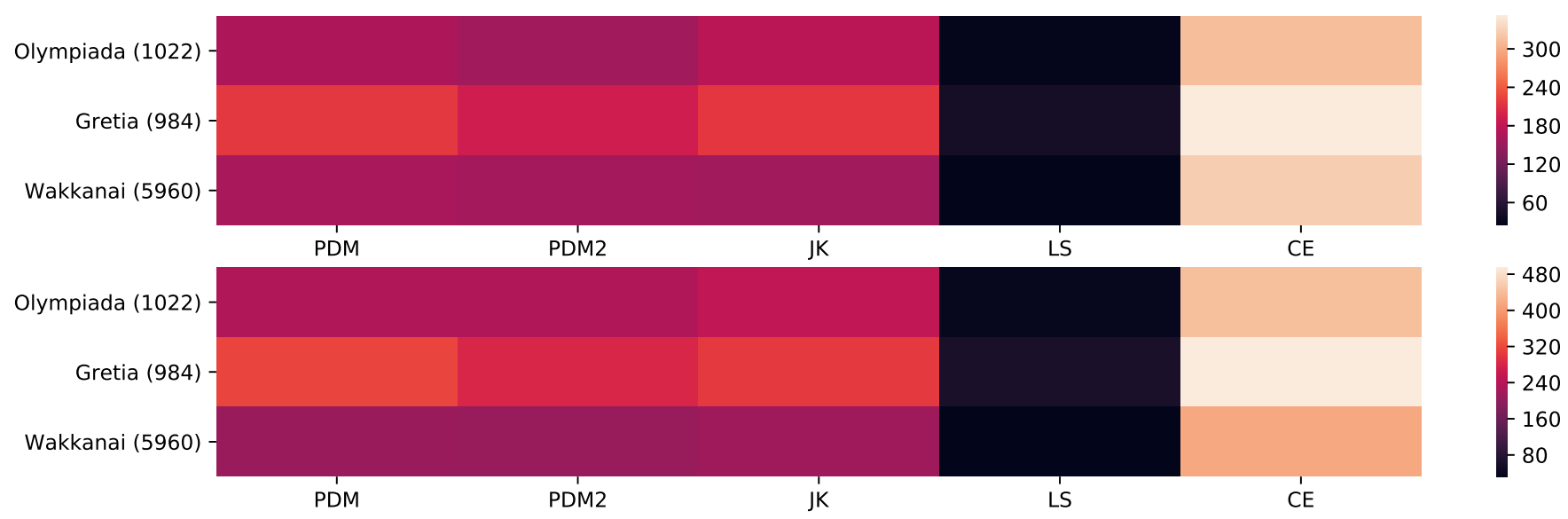

Figure 14. Calculation time in seconds for Olympiada (1022), Gretia (984) and Wakkanai (5960). Top panel: searching by periods with $p_{\text {step }}=10^{-4} \mathrm{~h}$ and searching range from 2 to $40 \mathrm{~h}$. Bottom panel: searching by frequencies with $f_{\text {step }}=10^{-6} \mathrm{~h}^{-1}$ and searching range from 0 to $0.5 \mathrm{~h}^{-1}$. 


\subsection{Error Analysis}

There are certain errors in the recording of parameters such as diameter and albedo in LCDB and DAMIT (see Table 8), so the above research results also have errors.

Table 8. Data error in DAMIT and LCDB $[25,26]$.

\begin{tabular}{cc}
\hline Index & Error Size \\
\hline Diameter & error is around $20 \%$ or more \\
Albedo & rounded to two decimal places \\
Absolute magnitude & accurate to two decimal places \\
Time span & accurate to 4 decimal places \\
Number of observation points & - \\
Search step size & depends on the specific situation \\
Observation time of & light curve JD epoch: accurate to six decimal places \\
Brightness & accurate to six decimal places \\
\hline
\end{tabular}

\section{Discussion}

Through the comparison of five different period extraction algorithms, we found that the accuracy of these five algorithms is not very different, but relatively speaking, the performance of PDM is better, followed by LS. However, after our comparison results, we found that the conditional entropy (CE) is not better than other algorithms.

By comparing six different asteroid parameters, we can draw the following conclusions:

(1) Absolute magnitude

The period extraction effect of various algorithms is negatively correlated with the magnitude. When the magnitude is small, the difference between various algorithms is not big, but the period of successful recovery ratio of PDM and LS is slightly better than other algorithms.

(2) Number of observations

When the number of observations is greater than one, the influence of various algorithms does not strongly depend on the number of sample points (only the third figure can be seen). However, when the number of sample points is less than $\exp (7)$, the results of PDM and LS are significantly better than other algorithms.

(3) Observation time span

As the observation time increases, the obtained asteroid light curve data is more abundant and reliable, so the percentage of successful periodic extraction is higher. From Figure 7, we find that the extraction effect of various algorithms is not bad. When the time span of observation is When it is less than $\exp (12)$, the extraction effect of $C E$ is relatively poor, but when the time span is greater than $\exp (12)$, the extraction effect of CE is the best.

(4) Albedo

By observing the albedo data, it is obvious that the effects of various period extraction algorithms do not show a certain regularity, and the ratio of successful recovery is almost irrelevant to the asteroid albedo. In general, the effect of PDM is better than other algorithms.

(5) Asteroid diameter

When the diameter is between $\exp (0) \mathrm{km}$ and $\exp (4) \mathrm{km}$, the impact on various algorithms does not fluctuate significantly. However, when the diameter is greater than $\exp (4) \mathrm{km}$, the proportion of asteroids that are successfully periodically recovered increases with the increase in diameter. It is obvious that the PDM and LS algorithms have the best effect, and the $\mathrm{CE}$ algorithm is relatively inferior to other algorithms.

(6) Search step By comparing different search step lengths, we find that the percentage of successful recovery cycles for $\mathrm{CE}$ is significantly weaker than other algorithms, while the effect of the other four algorithms is not much different. 
(7) Search period and search step length

By comparing the search period and search step size of the five algorithms, we found that if the search period range is $2-40 \mathrm{~h}$, the period search step size is $10^{-4}$ and $10^{-5}$, but when the report period is less than $10 \mathrm{~h}$, Search by frequency has better performance than the period search of each algorithm, and the effects of various algorithms after that have little difference. If the periodic search range is expanded to $2-750 \mathrm{~h}$, and $10^{-3} \mathrm{~h}$ is used as the matching criterion, it is clear that the effect of searching by periods is comparable to that of searching by frequencies at longer periods, however, this searching strategy works very poorly at short periods.

(8) Running speed

By observing the period extraction time of the five algorithms, we arrange the period extraction time of these five algorithms in ascending order of time, which are LS, $\mathrm{PDM}, \mathrm{JK}, \mathrm{PDM} 2, \mathrm{CE}$, and the extraction time of $\mathrm{CE}$ is about 2-5 times of the other four algorithms.

Further studies, which focus on the effects of some other promising algorithms for extracting the light curve periods using large amounts of light curve data from asteroids, will need to be undertaken. For example, we intend to apply these different period extraction algorithms to different asteroid campaigns like Gaia-GOSA.

\section{Related Work}

In order to facilitate astronomy enthusiasts to further explore the light curve, we recommend a related software-LAIA (Laboratory for Astronomical Image Analysis). It is a software package specially used to process astronomical images, which is often used to process images obtained by Baker Observatory and other national and international observatories. Some application cases of LAIA are shown below:

(1) Garcia-Melendo and Clement [43] have used LAIA to perform image cleaning and photometric analysis on RR Lyrae star NSV 09295, and accurately obtained its brightness changes at different times. After evaluation, it is found that the software's error in the magnitude analysis of the variable star is only $0.03 \mathrm{mag}$.

(2) LAIA has been used by Juan-Samsó et al. [44] to process the observational data of HIP 7666 which is one of the new variable stars discovered by the Hipparcos mission. Finally, it is discovered that HIP7666 is a new member of detached eclipsing binary systems.

(3) Using LAIA in SW1 (29P/Schwassmann-Wachmann 1), Trigo-Rodríguez et al. [45] succeeded in obtaining high-precision star luminosity, reducing the corresponding spatial scale to between 0.8 and $1.9 \mathrm{arc} \mathrm{sec/pixel.} \mathrm{Finally,} \mathrm{after} \mathrm{a} \mathrm{series} \mathrm{of} \mathrm{data}$ measurements, no signs of a clear periodicity in the outburst occurrence has been found, thus confirming the unpredictability of the activity of this comet.

In addition, the Google Brain team and Andrew Vanderburg of the University of Texas at Austin [46] have collaborated to build a CNN (Convolutional Neural Network) that can search for planets in the low signal-noise radio results. The neural network can be trained to distinguish planets by using about 15,000 Kepler signals (3500 are confirmed planets), manually checked and classified by humans. It is advisable to have a try.

\section{Conclusions}

In this paper, we have used the overall DAMIT data set to test and study the effects of several periodic analysis methods on asteroid data. The results show that:

(1) A minimum step size of $f_{\text {step }}=0.000001 \mathrm{~h}^{-1}$ in searching by frequencies is sufficient;

(2) The recommended method is searching by frequencies if the range of searching has not been determined. If the fine-grained search is proposed, searching by periods is more suitable;

(3) If the light curve data is in intensity units and made up of separate blocks observed by different observers, telescopes or filters, it is a good choice to make min-max 
normalization for the data in one block when the change of the phase angle is in the range of one degree;

$\delta \phi_{\max }=8500 / 50$ in Equation (6) is appropriate to be a criterion for the periodic recovery using the light curve data of asteroids which have the median time span of 134,615.14 h;

(5) Among all the steps, although the performance of all the algorithms varies little, PDM performs better, followed by LS, while CE is not better than the others under certain conditions. However, the most appropriate algorithm still needs to be determined based on the parameters of the observed object.

(6) LS is the fastest algorithm among these five algorithms, because it is not based on binning.

In Section 5, using the comparison of the performance against different parameters, we can draw a conclusion that when the parameters of the object itself make it difficult to be observed such as fainter absolute magnitude or shorter diameter, the accuracy of the calculated period can be improved by increasing the observation time, the number of observations and reducing the error and noise.

Author Contributions: Conceptualization, B.J.; methodology, T.S. and L.C.; software, T.S. and L.W.; validation, Y.L. and L.W.; formal analysis, X.F. and P.Z.; investigation, X.F.; resources, B.J.; writingoriginal draft preparation, L.W. and Y.L.; writing-review and editing, T.S. and L.W.; visualization, P.Z.; supervision, B.J.; project administration, B.J.; funding acquisition, B.J. All authors have read and agreed to the published version of the manuscript.

Funding: This paper is supported by Shandong Provincial Natural Science Foundation, China (No. ZR2020MA064).

Data Availability Statement: https://sbnarchive.psi.edu/pds4/non_mission/ast-lightcurve-database_ V4_0/data/ accessed on 1 November 2021, https:/ / astro.troja.mff.cuni.cz/projects/damit/ exports accessed on 1 November 2021.

Acknowledgments: We would like to dedicate the paper to Yang Liu, Shuaicong Yu, Xingzhu Wang, Xin Zhou, Jingyu Zhang, Shuting Wang and Xiaoming Kong who have offered us tremendous assistance. Additionally, we are grateful to the anonymous referee who made valuable suggestions to help improve the paper. The Database of Asteroid Models from Inversion Techniques (DAMIT) is a database of three-dimensional asteroid models that were derived from light curve data. The Asteroid Lightcurve Database (LCDB) lists asteroid lightcurve parameters and other information. We acknowledge the use of light curve data from DAMIT and asteroid information from LCDB. This research made use of astropy ${ }^{4}$, a community-developed core Python package for Astronomy and plotypus ${ }^{5}$, a Python library and command line utility for manipulating and plotting stellar lightcurves. We are grateful to those who supplied the codes for our research.

Conflicts of Interest: The authors declare no conflict of interest. The funders had no role in the design of the study; in the collection, analyses, or interpretation of data; in the writing of the manuscript, or in the decision to publish the results.

\section{Notes}

http:/ / www.minorplanetobserver.com/MPOSoftware/MPOCanopus.htm/ accessed on 1 November 2021.

https: / / astro.troja.mff.cuni.cz/projects/damit/ accessed on 1 November 2021.

http:/ / www.MinorPlanet.info/lightcurvedatabase.html accessed on 1 November 2021.

https:/ / www.astropy.org/ accessed on 1 November 2021.

https:/ / astroswego.github.io/plotypus/ accessed on 1 November 2021.

\section{References}

1. Buchheim, R.K. Methods and Lessons Learned Determining the H-G Parameters of Asteroid Phase Curves. Soc. Astron. Sci. Annu. Symp. 2010, 29, 101-115.

2. Dermott, S.F.; Harris, A.W.; Murray, C.D. Asteroid rotation rates. Icarus 1984, 57, 14-34. [CrossRef]

3. Binzel, R.P. Collisional evolution in the Eos and Koronis asteroid families: Observational and numerical results. Icarus 1988, 73, 303-313. [CrossRef] 
4. An, T.; Wang, J.Y.; Lu, X.L.; Lao, B.Q.; Wei, Y.H.; Dong, D.Q.; Lu, Y.; Wu, X.C. Review of Periodicity Searching Algorithms of Astronomical Light Curves (Chinese). Prog. Astron. 2016, 34, 74-93. [CrossRef]

5. Lowry, S.C.; Fitzsimmons, A.; Pravec, P.; Vokrouhlický, D.; Boehnhardt, H.; Taylor, P.A.; Margot, J.L.; Galád, A.; Irwin, M.; Irwin, J.; et al. Direct Detection of the Asteroidal YORP Effect. Science 2007, 316, 272. [CrossRef] [PubMed]

6. Butkiewicz-Bak, M.; Kwiatkowski, T.; Bartczak, P.; Dudziński, G.; Marciniak, A. Statistical analysis of the ambiguities in the asteroid period determinations. Mon. Not. R. Astron. Soc. 2017, 470, 1314-1320. [CrossRef]

7. Benishek, V.; Papini, R. Rotation Period Determination for 671 Carnegia. Minor Planet Bull. 2014, 41, $261-262$.

8. Szabó, R.; Pál, A.; Sárneczky, K.; Szabó, G.M.; Molnár, L.; Kiss, L.L.; Hanyecz, O.; Plachy, E.; Kiss, C. Uninterrupted optical light curves of main-belt asteroids from the K2 mission. Astron. Astrophys. 2016, 596, A40. [CrossRef]

9. Lomb, N.R. Least-Squares Frequency Analysis of Unequally Spaced Data. Astrophys. Space Sci. 1976, 39, 447-462. [CrossRef]

10. Scargle, J.D. Studies in astronomical time series analysis. II. Statistical aspects of spectral analysis of unevenly spaced data. Astrophys. J. 1982, 263, 835-853. [CrossRef]

11. Stellingwerf, R.F. Period determination using phase dispersion minimization. Astrophys. J. 1978, 224, 953-960. [CrossRef]

12. Wang, Y.B.; Wang, X.B. New CCD photometry of asteroid (1028) Lydina. Res. Astron. Astrophys. 2012, 12, 1714-1722. [CrossRef]

13. Prokof'eva, V.V.; Karachkina, L.G. Photometric and Colorimetric Observations of Asteroid 423 Diotima and Their Analysis. Sol. Syst. Res. 2004, 38, 108-119. [CrossRef]

14. Jurkevich, I. A Method of Computing Periods of Cyclic Phenomena. Astrophys. Space Sci. 1971, 13, 154-167. [CrossRef]

15. Reshetnyk, V.; Godunova, V.; Andreev, M.; Zhilyaev, B. Physical studies of near-Earth asteroids at the Terskol Observatory. In Asteroids, Comets, Meteors 2014; Muinonen, K., Penttilä, A., Granvik, M., Virkki, A., Fedorets, G., Wilkman, O., Kohout, T., Eds.; University of Helsinki: Helsinki, Finland, 2014; p. 439.

16. Sheppard, S.S.; Lacerda, P.; Ortiz, J.L. Photometric Lightcurves of Transneptunian Objects and Centaurs: Rotations, Shapes, and Densities. In The Solar System beyond Neptune; Barucci, M.A., Boehnhardt, H., Cruikshank, D.P., Morbidelli, A., Dotson, R., Eds.; The University of Arizona Press: Tucson, AZ, USA, 2008; p. 129.

17. Harris, A.W.; Young, J.W.; Bowell, E.; Martin, L.J.; Millis, R.L.; Poutanen, M.; Scaltriti, F.; Zappala, V.; Schober, H.J.; Debehogne, H.; et al. Photoelectric observations of asteroids 3, 24, 60, 261, and 863. Icarus 1989, 77, 171-186. [CrossRef]

18. Riccioli, D.; Blanco, C.; Cigna, M. Rotational periods of asteroids II. Planet. Space Sci. 2001, 49, 657-671. [CrossRef]

19. Heck, A.; Manfroid, J.; Mersch, G. On period determination methods. Astron. Astrophys. Suppl. Ser. 1985, 59, 63-72.

20. Swingler, D.N. A comparison of the Fourier, Jurkevich, and Stellingwerf methods of period estimation. Astron. J. 1989, 97, $280-287$. [CrossRef]

21. Schwarzenberg-Czerny, A. Optimum Period Search: Quantitative Analysis. Astrophys. J. 1999, 516, 315-323. [CrossRef]

22. Graham, M.J.; Drake, A.J.; Djorgovski, S.G.; Mahabal, A.A.; Donalek, C.; Duan, V.; Maker, A. A comparison of period finding algorithms. Mon. Not. R. Astron. Soc. 2013, 434, 3423-3444. [CrossRef]

23. Graham, M.J.; Drake, A.J.; Djorgovski, S.G.; Mahabal, A.A.; Donalek, C. Using conditional entropy to identify periodicity. Mon. Not. R. Astron. Soc. 2013, 434, 2629-2635. [CrossRef]

24. Harris, A.W.; Pravec, P.; Galád, A.; Skiff, B.A.; Warner, B.D.; Világi, J.; Gajdoš, Š.; Carbognani, A.; Hornoch, K.; Kušnirák, P.; et al. On the maximum amplitude of harmonics of an asteroid lightcurve. Icarus 2014, 235, 55-59. [CrossRef]

25. Durech, J.; Sidorin, V.; Kaasalainen, M. DAMIT: A database of asteroid models. Astron. Astrophys. 2010, 513, A46. [CrossRef]

26. Warner, B.D.; Harris, A.W.; Pravec, P. The asteroid lightcurve database. Icarus 2009, 202, 134-146. [CrossRef]

27. Ferraz-Mello, S. Estimation of Periods from Unequally Spaced Observations. Astron. J. 1981, 86, 619. [CrossRef]

28. Foster, G. The Cleanest Fourier Spectrum. Astron. J. 1995, 109, 1889. [CrossRef]

29. Foster, G. Wavelets for period analysis of unevenly sampled time series. Astron. J. 1996, 112, 1709. [CrossRef]

30. Stellingwerf, R.F. Period Determination of RR Lyrae Stars. In RR Lyrae Stars, Metal-Poor Stars, and the Galaxy; McWilliam, A., Ed.; The Observatories of the Carnegie Institution of Washington: Pasadena, CA, USA, 2011; Volume 5, p. 47.

31. Zappala, V.; Cellino, A.; Barucci, A.M.; Fulchignoni, M.; Lupishko, D.F. An analysis of the amplitude-phase relationship among asteroids. Astron. Astrophys. 1990, 231, 548-560.

32. McWhirter, P.R.; Wright, S.; Steele, I.A.; Al-Jumeily, D.; Hussain, A.; Fergus, P. A Dynamic, Modular Intelligent-Agent Framework for Astronomical Light Curve Analysis and Classification. In Intelligent Computing Theories and Application; Lecture Notes in Computer Science; Springer: Cham, Switzerland, 2016; Volume 9771, pp. 820-831. [CrossRef]

33. Astropy Collaboration; Robitaille, T.P.; Tollerud, E.J.; Greenfield, P.; Droettboom, M.; Bray, E.; Aldcroft, T.; Davis, M.; Ginsburg, A.; Price-Whelan, A.M.; et al. Astropy: A community Python package for astronomy. Astron. Astrophys. 2013, 558, A33. [CrossRef]

34. Price-Whelan, A.M.; Sipőcz, B.M.; Günther, H.M.; Lim, P.L.; Crawford, S.M.; Conseil, S.; Shupe, D.L.; Craig, M.W.; Dencheva, N.; Ginsburg, A.; et al. The Astropy Project: Building an Open-science Project and Status of the v2.0 Core Package. Astron. J. 2018, 156, 123. [CrossRef]

35. Hartmann, W.; Larson, S. Angular momenta of planetary bodies. Icarus 1967, 7, 257-260. [CrossRef]

36. Vio, R.; Diaz-Trigo, M.; Andreani, P. Irregular time series in astronomy and the use of the Lomb-Scargle periodogram. Astron. Comput. 2013, 1, 5-16. [CrossRef]

37. Schwarzenberg-Czerny, A. Fast and Statistically Optimal Period Search in Uneven Sampled Observations. Astrophys. J. 1996, 460, L107. [CrossRef] 
38. Debosscher, J.; Sarro, L.M.; Aerts, C.; Cuypers, J.; Vandenbussche, B.; Garrido, R.; Solano, E. Automated supervised classification of variable stars. I. Methodology. Astron. Astrophys. 2007, 475, 1159-1183. [CrossRef]

39. Richards, J.W.; Starr, D.L.; Miller, A.A.; Bloom, J.S.; Butler, N.R.; Brink, H.; Crellin-Quick, A. Construction of a Calibrated Probabilistic Classification Catalog: Application to 50k Variable Sources in the All-Sky Automated Survey. Astrophys. J. Suppl. Ser. 2012, 203, 32. [CrossRef]

40. Oluseyi, H.M.; Becker, A.C.; Culliton, C.; Furqan, M.; Hoadley, K.L.; Regencia, P.; Wells, A.J.; Ivezic, Ž.; Jones, R.L.; Krughoff, K.S.; et al. Simulated LSST Survey of RR Lyrae Stars throughout the Local Group. Astron. J. 2012, 144, 9. [CrossRef]

41. Dubath, P.; Rimoldini, L.; Süveges, M.; Blomme, J.; López, M.; Sarro, L.M.; De Ridder, J.; Cuypers, J.; Guy, L.; Lecoeur, I.; et al. Random forest automated supervised classification of Hipparcos periodic variable stars. Mon. Not. R. Astron. Soc. 2011, 414, 2602-2617. [CrossRef]

42. Polakis, T. Discovery and Period Analysis of Seven Variable Stars. J. Am. Assoc. Var. Star Obs. (JAAVSO) $2019,47,117$.

43. Garcia-Melendo, E.; Clement, C.M. NSV 09295: A double-mode RR lyrae variable. Astron. J. 1997, 114, 1190-1194. [CrossRef]

44. Juan-Samsó, J.; Vidal-Sáinz, J.; Lampens, P.; García-Melendo, E.; Gómez-Forrellad, J.; Wils, P. Detection of a classical $\delta$ Scuti star in the new eclipsing binary system HIP 7666. Astron. Astrophys. 2005, 434, 1063-1068.

45. Trigo-Rodríguez, J.; García-Melendo, E.; Davidsson, B.; Sánchez, A.; Rodríguez, D.; Lacruz, J.; de Los Reyes, J.; Pastor, S. Outburst activity in comets-I. Continuous monitoring of comet 29P/Schwassmann-Wachmann 1. Astron. Astrophys. 2008, 485, 599-606. [CrossRef]

46. Shallue, C.J.; Vanderburg, A. Identifying Exoplanets with Deep Learning: A Five-planet Resonant Chain around Kepler-80 and an Eighth Planet around Kepler-90. Astron. J. 2018, 155, 94. [CrossRef] 